PROCEEDINGS OF THE

AMERICAN MATHEMATICAL SOCIETY

Volume 129, Number 10, Pages 3121-3127

S 0002-9939(01)05913-5

Article electronically published on March 29, 2001

\title{
SUBSPACES OF KNOT SPACES
}

\author{
CRAIG BENHAM, XIAO-SONG LIN, AND DAVID MILLER
}

(Communicated by Ronald A. Fintushel)

\begin{abstract}
The inclusion of the space of all knots of a prescribed writhe in a particular isotopy class into the space of all knots in that isotopy class is a weak homotopy equivalence.
\end{abstract}

\section{INTRODUCTION}

In $[\mathrm{BM}]$, it is shown that the set of knots in any fixed isotopy class that have a prescribed writhe is path-connected. This result can be interpreted in the following manner. Let

$$
\mathcal{S}_{w} \stackrel{i^{\mathcal{S}}}{\longrightarrow} \mathcal{M}_{c}
$$

represent the inclusion of the space $\mathcal{S}_{w}$ of all knots of a prescribed writhe $w$ into the space $\mathcal{M}_{c}$ of all knots in some isotopy class $c$. Then the results of [BM] can be understood as stating that this map induces an isomorphism on $\pi_{0}$. In this paper we prove that this inclusion is a weak homotopy equivalence, inducing isomorphisms on $\pi_{n}$ for all $n$.

The proof that $\left(i^{\mathcal{S}}\right)_{*}: \pi_{n}\left(\mathcal{S}_{w}\right) \rightarrow \pi_{n}\left(\mathcal{M}_{c}\right)$ is an isomorphism uses an extension of the methods of $[\mathrm{BM}]$.

\section{Notation, DEFinitions AND STAtement of theorems}

Let $S^{1}=[0,1] / 0 \sim 1$. We define a closed space curve to be a smooth $\left(C^{1}\right)$ embedding

$$
\gamma: S^{1} \hookrightarrow \mathbb{R}^{3} .
$$

In general, we stipulate that $\gamma^{\prime}(s) \neq 0$. The image of the map $\gamma$ is referred to as a smooth knot.

Definition 2.1. The writhe of the embedding $\gamma$ is given by

$$
\mathbf{W r}(\gamma)=\iint_{\gamma\left(S^{1}\right) \times \gamma\left(S^{1}\right)-\Delta} d A
$$

Received by the editors June 28, 1999 and, in revised form, March 6, 2000.

1991 Mathematics Subject Classification. Primary 57M25, 53A04; Secondary 92B05.

Key words and phrases. Knots, writhe, DNA topology, knot spaces.

The first author was supported in part by NIH Grant RO1-GM47012.

The second author was supported in part by NSF Grant DMS-9704726.

The third author was supported in part by NSF Grant DMS-9732675. 
where $d A$ is the pull back of the area element on the unit sphere under the map which assigns to every pair $\left(\gamma(s), \gamma\left(s^{\prime}\right)\right) \in \gamma\left(S^{1}\right) \times \gamma\left(S^{1}\right)-\Delta$ the unit vector from $\gamma(s)$ to $\gamma\left(s^{\prime}\right)$.

By definition, the writhe is a geometric property of the image of $\gamma$, a property of the knot. In particular, it is independent of parameterization. We can thus refer alternatively to the writhe, $\operatorname{Wr}(K)$, of the knot $K$. We let $\mathcal{M}_{c}$ denote an arbitrary component of the space of knots and $\mathcal{S}_{\omega}$ denote the subset of $\mathcal{M}_{c}$ of curves with a particular writhe, $\omega$.

Theorem 2.1. The induced map

$$
\left(i^{\mathcal{S}}\right)_{*}: \pi_{n}\left(\mathcal{S}_{w}, \gamma_{0}\right) \rightarrow \pi_{n}\left(\mathcal{M}_{c}, \gamma_{0}\right)
$$

is an isomorphism for all $n$, where the basepoint $\gamma_{0}$ is a smooth knot of writhe $\omega$.

The proof of Theorem 2.1 will extend the results and proofs of [BM], which can be understood as Theorem 2.1 in the case $n=0$. In particular, we need the following theorem of Fuller.

Theorem 2.2 ([F1, Fuller]). The unit tangents $T(s)$ to a smooth, closed space curve, $\gamma$, trace out, if their starting points are translated to the origin, a closed curve on the unit sphere. Let $A$ be the area on the unit sphere enclosed by this curve. Then

$$
1+\mathbf{W r}(\gamma) \equiv \frac{1}{2 \pi} \text { A, modulo } 2 .
$$

\section{Construction OF FiXed-Writhe FAMilies of EMBEDdings}

In this section we will generalize the results of $[\mathrm{BM}]$ in two different settings in order to analyze the higher connectivity of the sets of fixed writhe within components of the space of knots.

First consider any family of embedded curves

$$
\gamma_{x}: S^{1} \hookrightarrow \mathbb{R}^{3}, \quad x \in\left(S^{n}, x_{0}\right),
$$

indexed by the based $n$-dimensional sphere such that the map

$$
\Gamma: S^{1} \times S^{n} \rightarrow \mathbb{R}^{3} \times S^{n}, \quad \Gamma(s, x) \stackrel{\text { def. }}{=}\left(\gamma_{x}(s), x\right),
$$

is continuous. From $\Gamma$ we can construct

$$
\Psi: S^{n} \rightarrow \mathcal{M}_{c}, \quad \Psi(x) \stackrel{\text { def. }}{=} \gamma_{x} .
$$

Let

$$
\omega=\mathbf{W r}\left(\gamma_{x_{0}}\right)
$$

Our goal is to construct a family of embeddings $\bar{\gamma}_{x}$ such that $\mathbf{W r}\left(\bar{\gamma}_{x}\right)=\omega$ for all $x \in S^{n}$. To accomplish this goal, we must first alter each embedding in the original family in order to allow for a correction in the writhe.

We choose once and for all a point $s_{0} \in S^{1}, 0 \neq s_{0} \neq 1$. Let $T_{x}\left(s_{0}\right)$ be the unit tangent to $\gamma_{x}$ at $s_{0}$. We reparameterize the maps $\gamma_{x}$ such that for each $x \in S^{n}$, there is an interval $\left[s_{1}, s_{2}\right]$ containing $s_{0}$ and for any $s \in\left[s_{1}, s_{2}\right], \gamma_{x}(s)=\gamma_{x}\left(s_{0}\right)$. This can be accomplished by reparameterizing $\Gamma$ so that it is constant on $\left[s_{1}, s_{2}\right] \times S^{n} \subset$ $S^{1} \times S^{n}$ and not on any larger closed set, i.e., $\Gamma^{-1}\left(\gamma_{x}\left(s_{0}\right)\right)=\left[s_{1}, s_{2}\right] \times I$. Then for each $x \in[0,1]$, let $B_{x, \epsilon} \subset \mathbb{R}^{3}$ be a closed ball of radius $\epsilon$ with $\gamma_{x}\left(s_{0}\right)$ at its center. $\epsilon$ can be chosen arbitrarily small, but is fixed for all $x$. Let $\phi_{x}: B_{x, \epsilon} \rightarrow B_{\epsilon} \hookrightarrow \mathbb{R}^{3}$ be 
a linear, co-ordinate chart for $B_{x, \epsilon}$, i.e., a translation map taking the point $\gamma_{x}\left(s_{0}\right)$ to the origin, and the ball $B_{x, \epsilon}$ to a ball $B_{\epsilon}$ of radius $\epsilon$ centered at the origin.

We construct for each $x$ a map $\sigma_{x}:\left(B_{\epsilon}-\{\mathbf{0}\}\right) \longrightarrow\left(B_{\epsilon}-\{\mathbf{0}\}\right)$ as follows:

$$
\sigma_{x}(z)= \begin{cases}{\left[\left(1-\frac{3|x|}{2}\right)|z|+\frac{3|x| \epsilon}{2}\right] \frac{z}{|z|},} & 0 \leq|x| \leq \frac{1}{3}, \\ {\left[\frac{1}{2}|z|+\frac{\epsilon}{2}\right] \frac{z}{|z|},} & |x| \geq \frac{1}{3} .\end{cases}
$$

These linear maps have a number of important properties. First note that $\sigma_{x_{0}}=$ $\mathbf{i d}_{B_{\epsilon}}$. Note also that for each $x,\left.\sigma_{x}\right|_{S_{\epsilon}}=\mathbf{i d}_{S_{\epsilon}}$. Most importantly, these maps have the property that they "push" the contents of $B_{\epsilon}-\{\mathbf{0}\}$ towards the boundary in a linear fashion, creating an "empty space" around the origin. When $x$ is close to $x_{0}$, the contents are not pushed as far.

We next assemble a map $\tilde{\sigma}: \mathbb{R}^{3} \rightarrow \mathbb{R}^{3}$ by

$$
\tilde{\sigma}_{x}(z)=\left\{\begin{array}{lr}
z, & z \in \mathbb{R}^{3}-\left(B_{x, \epsilon}-\left\{\gamma_{x}\left(s_{0}\right)\right\}\right), \\
\left(\phi_{x}^{-1} \circ \sigma_{x} \circ \phi_{x}\right)(z), & z \in B_{x, \epsilon}-\left\{\gamma_{x}\left(s_{0}\right)\right\} .
\end{array}\right.
$$

If, for $x \neq x_{0}$, we think of our $\operatorname{knot}, \operatorname{im}\left(\gamma_{x}\right)$, lying in $\mathbb{R}^{3}$, its image under $\tilde{\sigma}_{x}$ would no longer be a closed curve. There would be a gap between the two points where the curve touched a certain small sphere around $\gamma_{x}\left(s_{0}\right)$. To rectify this problem, we construct an altered isotopy,

$$
\tilde{\gamma}_{x}(s)=\left\{\begin{array}{l}
\left(\tilde{\sigma}_{x} \circ \gamma_{x}\right)(s), \quad s \in\left(S^{1}-\left[s_{1}, s_{2}\right]\right), \\
\phi_{x}^{-1}\left(\frac{2 \epsilon}{s_{2}-s_{1}}\left[s-\frac{s_{1}+s_{2}}{2}\right]\left(T_{x}\left(s_{0}\right)\right)\right), \quad s \in\left[s_{1}, s_{2}\right] .
\end{array}\right.
$$

The definition of $\tilde{\gamma}_{x}$ on $\left[s_{1}, s_{2}\right]$ has the effect of inserting a straight line segment in the gap left by $\tilde{\sigma}_{x}$, and connecting smoothly with $\tilde{\gamma}_{x}\left(s_{1}\right)$ and $\tilde{\gamma}_{x}\left(s_{2}\right)$ at the ends.

The final step in constructing a family of embeddings of fixed writhe is to replace the altered portion of the curves with helical segments which will provide the appropriate correction in the writhe. By Theorem 2.2, the writhe of a knot is determined modulo an integral indeterminacy by the area on the unit sphere enclosed by the tangent indicatrix, the curve that the unit tangents to the knot trace out. We will use this fact to construct a local correction to the altered isotopy above which will precisely fix the writhe throughout the isotopy. Let $M=\max _{x}\left(\mathbf{W r}\left(\tilde{\gamma}_{x}\right)\right)$ and $m=\min _{x}\left(\mathbf{W r}\left(\tilde{\gamma}_{x}\right)\right)$. Let $n$ be the smallest integer strictly greater than $M-m$. Let $w(x)=\omega-\mathbf{W r}\left(\tilde{\gamma}_{x}\right)$. Note that $|w(x)|<n$. We shall modify each embedding $\tilde{\gamma}_{x}: S^{1} \hookrightarrow \mathbb{R}^{3}$ on the interval $\left[s_{1}, s_{2}\right]$. Let

$$
s_{3}=s_{1}+\frac{s_{2}-s_{1}}{4}, \quad s_{4}=s_{2}-\frac{s_{2}-s_{1}}{4} .
$$

On $\left[s_{3}, s_{4}\right]$, and for each $x \in S^{n}$ we define the following helix function:

$$
\tau_{x}(s)=\left(r(x) \cos (C(x) s), r(x) \sin (C(x) s), p(x) \frac{n}{s_{4}-s_{3}}\left(s-\frac{s_{4}+s_{3}}{2}\right)\right),
$$


where

$$
\begin{aligned}
& C(x)=-\operatorname{sgn}(w(x)) \frac{2 \pi n}{s_{4}-s_{3}}, \\
& r(x)=S(x) \frac{\epsilon}{4 \pi n} \sqrt{\frac{2|w(x)|}{n}-\frac{w(x)^{2}}{n^{2}}}, \\
& p(x)=S(x) \frac{\epsilon}{2 n}\left(1-\frac{|w(x)|}{n}\right),
\end{aligned}
$$

and where $S(x)$ is a scale function given by

$$
S(x)= \begin{cases}3 x, & 0 \leq|x| \leq \frac{1}{3}, \\ 1, & |x|>\frac{1}{3} .\end{cases}
$$

Thus the image of $\tau_{x}$ is a helix with precisely $n$ turns. The pitch and radius of the helix are determined by $w(x)$, but each turn of the helix is contained in a ball of radius $S(x) \frac{\epsilon}{2 n}$. If $w(x)$ goes to zero, the helix degenerates into a vertical line of length $\frac{\epsilon}{2}$, from $\left(0,0,-\frac{\epsilon}{4}\right)$ to $\left(0,0, \frac{\epsilon}{4}\right)$. If $|w(x)|$ gets close to $n$, the pitch angle becomes small.

We must now connect the image of $\tau_{x}$ with the south and north poles of the sphere of radius $S(x) \frac{\epsilon}{2}$ centered at the origin. To do this we consider the plane spanned by the unit vectors

$$
\mathbf{v}_{1}=(0,0,1) \quad \text { and } \quad \mathbf{v}_{2}=\frac{\tau_{x}^{\prime}\left(s_{3}\right)}{\left|\tau_{x}^{\prime}\left(s_{3}\right)\right|}=\frac{\tau_{x}^{\prime}\left(s_{4}\right)}{\left|\tau_{x}^{\prime}\left(s_{4}\right)\right|}
$$

We then choose $\iota_{x}:\left[s_{1}, s_{3}\right] \hookrightarrow\left(B_{S(x) \frac{\epsilon}{2}}^{-}-B_{\left\|\tau_{x}\left(s_{3}\right)\right\|}^{-}\right)$to be any smooth curve in the $\mathbf{v}_{1}-\mathbf{v}_{2}$ plane such that

$$
\begin{aligned}
& \iota_{x}\left(s_{1}\right)=\left(0,0,-\frac{\epsilon}{2}\right), \quad \frac{\iota_{x}^{\prime}\left(s_{1}\right)}{\left|\iota_{x}^{\prime}\left(s_{1}\right)\right|}=(0,0,1), \\
& \iota_{x}\left(s_{3}\right)=\tau_{x}\left(s_{3}\right), \quad \frac{\iota_{x}^{\prime}\left(s_{3}\right)}{\left|\iota_{x}^{\prime}\left(s_{3}\right)\right|}=\frac{\tau_{x}^{\prime}\left(s_{3}\right)}{\left|\tau_{x}^{\prime}\left(s_{3}\right)\right|} .
\end{aligned}
$$

There are clearly many such curves, as we are merely requiring that $\iota$ be a curve on a plane with specified end-points and specified tangent directions at those endpoints. (In the special case where $\mathbf{v}_{1}=\mathbf{v}_{2}$, the situation is even simpler. We merely connect the two end-points with a vertical line.) Our next step is to connect $\tau_{x}$ to the north pole by defining $\xi_{x}:\left[s_{3}, s_{4}\right] \hookrightarrow\left(B_{S(x) \frac{\epsilon}{2}}^{+}-B_{\left\|\tau_{x}\left(s_{4}\right)\right\|}^{+}\right)$by

$$
\xi_{x}(s)=r\left(\iota_{x}\left(s_{3}-\left(s-s_{4}\right)\right)\right)
$$

where $r$ is reflection about the $x_{1} x_{2}$-plane. We now assemble a map $\eta_{x}:\left[s_{1}, s_{2}\right] \hookrightarrow$ $B_{S(x) \frac{\epsilon}{2}}$ as follows:

$$
\eta_{x}(s)= \begin{cases}\iota_{x}(s), & s_{1} \leq s<s_{3}, \\ \tau_{x}(s), & s_{3} \leq s \leq s_{4}, \\ \xi_{x}(s), & s_{4}<s<s_{2} .\end{cases}
$$


Let $\theta \in S O(3)$ be any rotation map which takes the unit vector $(0,0,1)$ to the unit vector $T_{x}\left(s_{0}\right)$. Finally, we assemble the map

$$
\bar{\gamma}_{x}(s)=\left\{\begin{array}{l}
\tilde{\gamma}_{x}(s), \quad s \in\left(S^{1}-\left[s_{1}, s_{2}\right]\right), \\
\phi_{x}^{-1}\left(\theta\left(\eta_{x}(s)\right)\right), \quad s \in\left[s_{1}, s_{2}\right] .
\end{array}\right.
$$

We shall prove that $\mathbf{W r}\left(\bar{\gamma}_{x}\right)=\omega$ for all $x \in S^{n}$. We use Theorem 2.2, comparing the area that the tangent indicatrix to $\bar{\gamma}_{x}$ traces out to that traced out by $\tilde{\gamma}_{x}$. The difference will be equal to $w(x)$. We first note that $\gamma_{x}$ was parameterized to be constant on the interval $\left[s_{1}, s_{2}\right]$, so that the tangent indicatrix curve is not welldefined. However, our analysis was conducted for $\tilde{\gamma}_{x}$. This curve behaved linearly on $\left[s_{1}, s_{2}\right]$. Thus the tangent indicatrix curve for $\tilde{\gamma}_{x}$ on the unit sphere, $\widetilde{T}_{x}(s)$, is constant on $\left[s_{1}, s_{2}\right]$. In defining $\bar{\gamma}$ we changed the behavior of the curve on the interval $\left[s_{1}, s_{2}\right]$ in a precise manner. The effect this has on the tangent indicatrix and the area it encloses can be easily determined. The intervals $\left[s_{1}, s_{3}\right]$ and $\left[s_{4}, s_{2}\right]$, determined respectively by $\iota_{x}$ and $\xi_{x}$, have closely related tangent behavior. As $s$ goes from $s_{1}$ to $s_{3}$, the tangent indicatrix will travel along some curve from $\bar{T}_{x}\left(s_{1}\right)$ to $\bar{T}_{x}\left(s_{3}\right)$. While $s$ goes from $s_{4}$ to $s_{2}$, the indicatrix of $\bar{T}_{x}(s)$ will travel back along the same curve. Thus, these intervals together do not contribute to the area enclosed by the tangent indicatrix. It only remains to examine the tangent indicatrix curve on the interval $\left[s_{3}, s_{4}\right]$. On this interval, $\bar{\gamma}_{x}$ is a helix which winds $n$ times, and by virtue of the definition of $\tau_{x}$ has pitch angle $\psi_{x}$ with the property that

$$
\sin \psi_{x}=1-\frac{w(x)}{n}
$$

which means that

$$
w(x)=n\left(1-\sin \psi_{x}\right) .
$$

But this last expression is precisely $n$ times the signed area of the cap on the unit sphere that the unit tangents to an $n$-turn helix of pitch angle $\psi_{x}$ trace out $n$ times. Thus the insertion of the helix $\tau_{x}$ contributes precisely $w(x)$ to the area traced out by the tangent indicatrix. (Since the writhe changes continuously through the isotopy, the indeterminacy in Theorem 2.2 does not arise.) Thus, we see that

$$
\mathbf{W r}\left(\bar{\gamma}_{x}\right)=\mathbf{W r}\left(\tilde{\gamma}_{x}\right)+w(x)=\omega .
$$

In the next setting to which we will generalize the results of $[\overline{\mathrm{BM}}]$, we consider families of embedded curves

$$
\lambda_{x}: S^{1} \hookrightarrow \mathbb{R}^{3}, \quad x \in\left(S^{n} \times I, x_{0}\right),
$$

such that

$$
\Lambda: S^{1} \times\left(S^{n} \times I\right) \rightarrow \mathbb{R}^{3} \times\left(S^{n} \times I\right), \quad \Lambda(s, x) \stackrel{\text { def. }}{=}\left(\lambda_{x}(s), x\right) .
$$

In addition, we stipulate that if $x \in\left(S^{n} \times\{0\}\right)$ or $x \in\left(S^{n} \times\{1\}\right)$, then $\mathbf{W r}\left(\lambda_{x}\right)=\omega$. Our goal is, again, to construct a family of embeddings $\bar{\lambda}_{x}$ such that $\operatorname{Wr}\left(\bar{\lambda}_{x}\right)=\omega$, for all $x \in\left(S^{n} \times I\right)$. From $\Lambda$, we can construct

$$
\Upsilon: S^{n} \times I \rightarrow \mathcal{M}_{c}, \quad \Upsilon(x) \stackrel{\text { def. }}{=} \lambda_{x} .
$$

The existence of the curves $\bar{\lambda}_{x}$ is proved with exactly the construction of $\mathrm{BM}$ ] crossed with $S^{n}$. 


\section{Proof of Theorem 2.1}

We first show that the map $\left(i^{\mathcal{S}}\right)_{*}$ is onto. We first define

$$
\bar{\Gamma}: S^{1} \times S^{n} \rightarrow \mathbb{R}^{3} \times S^{n}, \quad \bar{\Gamma}(s, x) \stackrel{\text { def. }}{=}\left(\bar{\gamma}_{x}(s), x\right),
$$

and

$$
\bar{\Psi}: S^{n} \rightarrow \mathcal{M}_{c}, \quad \bar{\Psi}(x) \stackrel{\text { def. }}{=} \bar{\gamma}_{x} .
$$

We define $\tilde{\Gamma}$ and $\tilde{\Psi}$ similarly. We then define a homotopy

$$
\Omega: S^{n} \times I \rightarrow \mathcal{M}_{c} \times I
$$

as follows. Let $\Omega_{0} \stackrel{\text { def. }}{=} \Psi$ and $\Omega_{1} \stackrel{\text { def. }}{=} \bar{\Psi}$. For $\frac{1}{2} \leq t \leq 1$ we define $\Omega_{t}$ to be $\bar{\Psi}$, except that in the definition of $\bar{\gamma}_{x}$, we replace $r(x)$ with $(2 t-1) r(x)$. This has the effect of shrinking the width of the inserted helix as $t$ decreases from 1 to $\frac{1}{2}$, so that $\Omega_{\frac{1}{2}}=\tilde{\Psi}$. For $0 \leq t<\frac{1}{2}$, we define $\Omega_{t}$ to be $\tilde{\Psi}$, except that in the definition of $\tilde{\gamma}_{x}$, we replace $\epsilon$ with $2 t \epsilon$. We then see that $\Omega$ is a homotopy between $\Psi$ and $\bar{\Psi}$.

If we take any element $y \in \pi_{n}\left(\mathcal{M}_{c}\right)$ and write $y=[\Psi]$, the existence of $\Omega$ implies that $y=[\bar{\Psi}]$. But since

$$
\bar{\Psi}: S^{n} \rightarrow \mathcal{S}_{w},
$$

we see that $y \in i_{*}^{\mathcal{S}}\left(\pi_{n}\left(\mathcal{S}_{w}\right)\right)$. Since the choice of $y$ was arbitrary, $i_{*}^{\mathcal{S}}$ is onto.

We now show that the map $\left(i^{\mathcal{S}}\right)_{*}$ is one-to-one. We define

$$
\bar{\Lambda}: S^{1} \times\left(S^{n} \times I\right) \rightarrow \mathbb{R}^{3} \times\left(S^{n} \times I\right), \quad \bar{\Lambda}(s, x) \stackrel{\text { def. }}{=}\left(\bar{\lambda}_{x}(s), x\right),
$$

and we define

$$
\bar{\Upsilon}:\left(S^{n} \times I\right) \rightarrow \mathcal{M}_{c}, \quad \bar{\Upsilon}(x) \stackrel{\text { def. }}{=} \bar{\lambda}_{x} .
$$

We define $\tilde{\Lambda}$ and $\tilde{\Upsilon}$ similarly. We define a homotopy

$$
\Phi:\left(S^{n} \times I\right) \times I \rightarrow \mathcal{M}_{c} \times I,
$$

as follows. For $\frac{1}{2} \leq t \leq 1$, let $\Phi_{t}$ be the same as $\bar{\Upsilon}$ except that in the definition of $\bar{\lambda}_{x}$, we replace $r(x)$ with $(2 t-1) r(x)$. Thus, $\Phi_{1}=\bar{\Upsilon}$ and $\Phi_{\frac{1}{2}}=\tilde{\Upsilon}$. For $0 \leq t<\frac{1}{2}$, we define $\Phi_{t}$ to be $\tilde{\Upsilon}$, except that in the definition of $\tilde{\lambda}_{x}$, we replace $\epsilon$ by $2 t \epsilon$. We then see that $\Phi$ is a homotopy between $\Upsilon$ and $\bar{\Upsilon}$.

If we take two elements $y, z \in \pi_{n}\left(\mathcal{S}_{w}\right)$, represented respectively by $\Theta$ and $\Sigma$, such that

$$
\left(i^{\mathcal{S}}\right)_{*}(y)=\left(i^{\mathcal{S}}\right)_{*}(z)
$$

there exists a homotopy which we will denote by $\Lambda$, between $\Theta$ and $\Sigma$. But since $\Lambda$ can be homotoped to $\bar{\Lambda}$ via $\Phi$ above, we see that $\bar{\Lambda}$ is a homotopy between $\Theta$ and $\Sigma$ within $\mathcal{S}_{w}$. Thus $y=z$ and $\left(i^{\mathcal{S}}\right)_{*}$ is one-to-one.

\section{REMARKS}

This work was motivated by the role of the writhe in DNA topology. For a closed DNA domain, the writhe of the molecular backbone is a quantity which is strongly determined by chemical stresses on the domain. So results on the writhe as a functional on knot space may have important applications to the understanding of DNA structure and function. 
It should be noted that the Smale conjecture, proved in [H1], implies that the spaces $\mathcal{M}_{c}$ are in fact orbit spaces of certain $K(\pi, 1)$-spaces under an action of $\operatorname{Diff}\left(S^{1}\right)$. This result is proved explicitly in [H2].

It is a result of Gluck and Pan GP that the set of knots in any fixed isotopy class with non-vanishing curvature and a prescribed self-linking number is pathconnected. In a future paper, we will show that the inclusion of the set of all such knots into the set of all knots in a particular isotopy class is also a weak homotopy equivalence.

\section{REFERENCES}

[BM] C. Benham and D. Miller, Fixed-writhe isotopies and the topological conservation law for closed, circular DNA, Journal of Knot theory and its Ramifications, 5 (1996), 859-866. MR 98b:57019

[F1] F.B. Fuller, Decomposition of the linking number of a closed ribbon: A problem from molecular biology, Proc. Natl. Acad. Sci. USA, 75 (1978), 3557-3561. MR 58:9367

[F2] F.B. Fuller, The writhing number of a Space Curve, Proc. Natl. Acad. Sci. USA, 68 (1971), 815-819. MR 43:3928

[GP] H. Gluck and L.H. Pan, Knot theory in the presence of curvature I, University of Pennsylvania preprint, 1994.

[H1] A. Hatcher, A proof of the Smale conjecture, Ann. of Math. 117 (1983), 553-607. MR 85c:57008

[H2] A. Hatcher, Spaces of Knots, Cornell University Preprint, 1999, http://www.math.cornell.edu/ hatcher.

[P] W.F. Pohl, the self-linking number of a closed space curve, Journal of Mathematics and Mechanics, 17 (1968), 975-985. MR 36:5827

Department of Biomathematical Sciences, Mount Sinai School of Medicine, One Gustave Levy Place, New York, New York 10029

E-mail address: BENHAM@msvax.mssm.edu

Department of Mathematics, University of California Riverside, Riverside, CaliforNIA 92521

E-mail address: xl@math.ucr.edu

Department of Mathematics, William Paterson University, Wayne, New Jersey 07470

E-mail address: damiller@pilot.njin.net 